\title{
An overview on wireless sensor networks and finding optimal location of nodes
}

\author{
Osamah Ibrahim Khalaf ${ }^{1}$, Bayan Mahdi Sabbar ${ }^{2}$ \\ College of Information Engineering, AI-Nahrain University, Baghdad, Iraq ${ }^{1,2}$
}

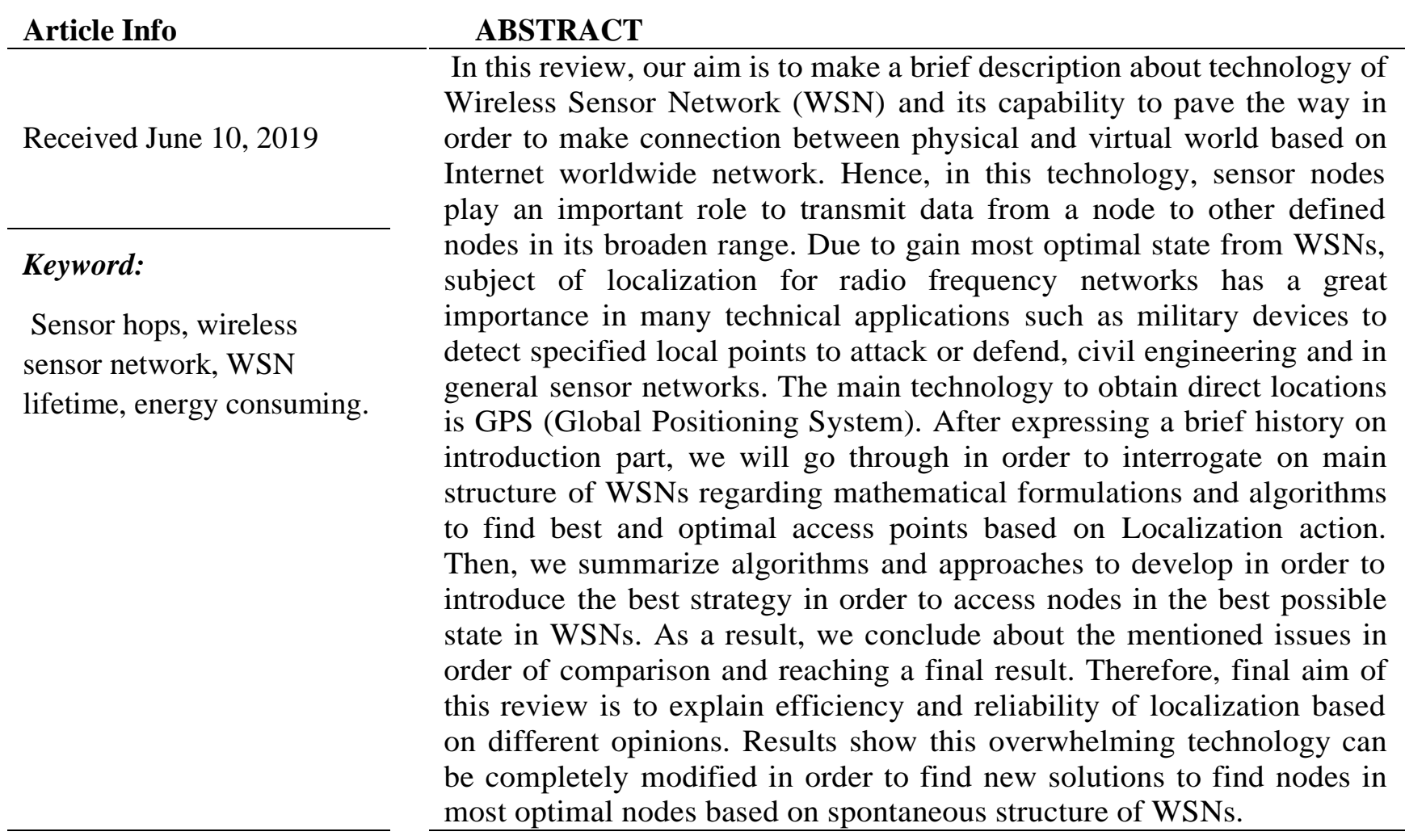

\section{Corresponding Author:}

Dr. Osamah Ibrahim Khalaf

AI-Nahrain University

College of Information Engineering

Email: usama.ibrahem@coie-nahrain.edu.iq

\section{Introduction}

Nowadays, due to fast progress of internet extension in all aspects of our lives, science and technology throughout the world, Intention of researchers and scientists has increased to find the best localization methods to optimize WSNs. Generally, WSNs are formed of a group of small, low-cost sensor nodes in order to communicate with each other in which they have been fitted by some restrictions in their memory, energy and processing capacity in wireless format [1-4].

Similarity of WSNs are like Wireless ad hoc networks at which they are related to wireless connectivity and automatic structure of networks such that sensor data are able to transport in wireless form. In fact, WSNs act as distributed autonomous sensors that can monitor or supervise physical and environmental 
circumstances. They can be all natural phenomena like temperature, sound, pressure, etc. in a way that transmit their data via formed network to a main location. An illustrative example of WSNs is given in Figure 1. A full comprehension of WSNs and its structure will be proposed in the next section.

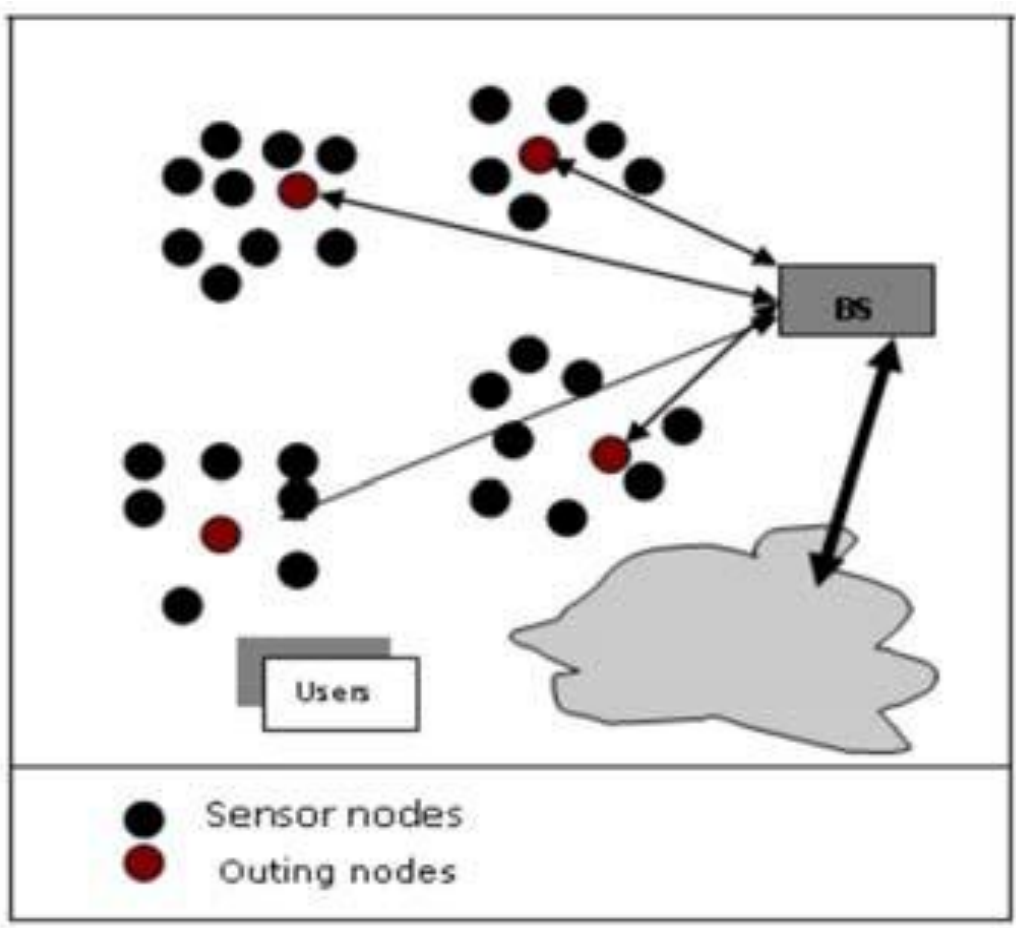

Figure 1. An illustrative structure of Wireless Sensor Networks

\section{Architecture of WSNs and sink nodes}

In this section, we will investigate on main structure of Wireless Sensor Networks based on specified sink nodes. Based on main concept of WSNs [5], sensor nodes by routing capabilities will be scattered in a sensor field. Also, according to location of sensor nodes, random deployment can be allowed in inaccessible areas including disaster relief operations or battlefields. Meanwhile, in order to distinguish between WSNs and wireless ad hoc networks, these characteristics will be featured:

1- Number of Sensor nodes in WSNs should be more than nodes in ad hoc networks.

2- Sensor nodes needs to be applied in a densely connectivity format, otherwise it will be confronted with redundancy in in the board of measurements.

3- Sensor nodes tend to failures for getting a powerful connectivity from other nodes.

4- Sensor nodes have restriction about power, computational capacities and memory.

A full illustrative explanation about Sink nodes has been demonstrated in Figure2. Each node communicates with its vicious node in any network of connections. Based on new designs by network engineers [5], multi-hop communication Technique in sensor networks is consumable to use power rather than single-hop ones that needs to be applied large number of densely sensor nodes as a traditional approach. One of constraints on sensor nodes is to use low level of power.WSNs are counted for Microelectronics devices and its big avatantage to use them is limited source of power. Power sources of WSNs are life time and also irreplaceable for some technical issues in engineering applications.Essentailly, WSN protocols focus on primary power preservation and single hope ones is usually forbidden to implement. 


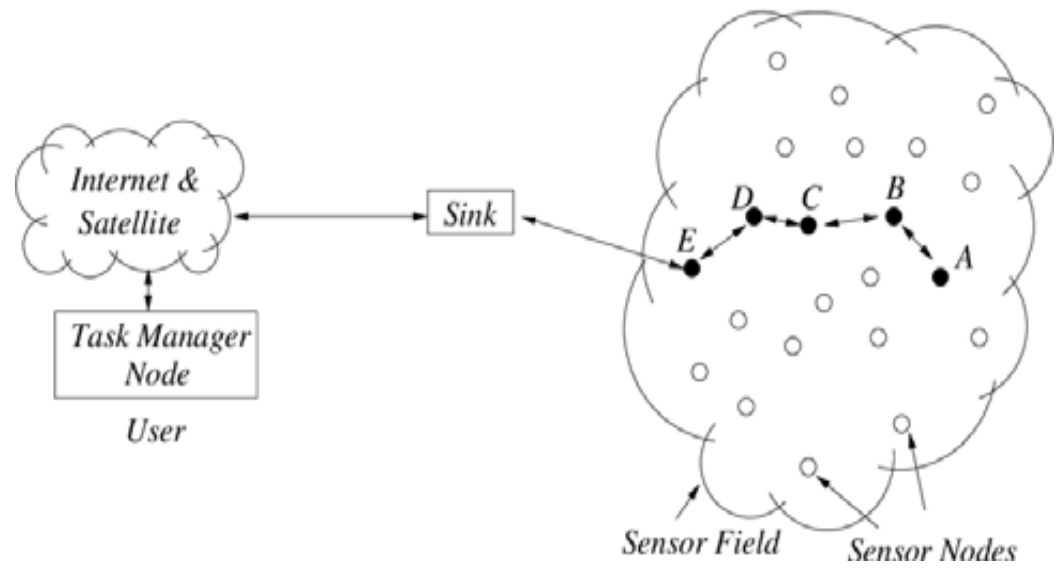

Figure 2. Sink node with scattered sensor nodes

\section{Localization Techniques and dependent parameters}

In this section, we investigate about optimal and competitive methods on localization of WSNs[6,7]. Now, we review some concepts and important parameters for localization. These parameters include:

1- Accuracy: This is an important parameter in order to estimate information of accurate location of sensor nodes. However, in practice it does not imply a guarantee to find accurate resulted information of wireless sensor nodes.

2- Cost: One of the most important factors to choose main structure of WSNs to implement is cost and expense of localization technique applied on sensor networks. Therefore, that is why we emphasize on optimal phrase to select suitable localization approach to develop on chosen sensor nodes in the defined network.

3- Power: This parameter also is assumed and used in computation process of WSNs. Power is considered as a restricted source in WSNs. Naturally, it would be better to choose technique in which it involves less computational operations in order to find optimal location of sensor nodes.

4- Node density: As we know in case study of WSNs, Certain localization methods are dependent to parameter of node density. If our choice be localization approach based on hop-count (number of nodes), then it needs a high node density of network.

5- Type of Nodes: This parameter can be chosen as static or dynamic. Based on definition, identical battery power source will be used to deploy static sensor nodes. But more battery power source is supposed to be used for mobile sensor nodes compared to static type.

Based on complete considerations that issued above, WSNs will be deployed similarly in ad-hoc state. So, there exist any information about nodes that have been located. Therefore, GPS is one of the used techniques to estimate position of sensor nodes for localization. But, in practice this technology is expensive to use and one of its disadvantages is working just in outdoor spots. Hence, it has been defined various optimal localization methods that are categorized as :

1- Anchor based and Anchor free method [8]

2- Range based and Range free

3-Centralized and Distributed

Anchors are defined as sensor nodes with location information. Their position is specified by means of GPS or launching anchors with certain coordinates. On the other hand, we are facing with non- anchor nodes in which their coordinates need to be estimated by localization approaches. Anchor based localization works with anchor nodes that are located and economical optimization is gained by using this method rather than 
GPS as mentioned about its disadvantages. Anchor Free localization deploys a relative location of sensor nodes that are considered to compute in their absolute location. In [9] a comparison between anchors based and anchor free methods has been discussed for using beacons as reference nodal points in anchor based rather than anchor free method. Hence, at fist in anchor based method, a set of reference points are identified then, after implementing a spatial relation with reference points, the process of estimating their own locations will be done. An illustrative schematic has been presented in Figure3.Range based methods get information about distance of vicious points and this distance will be estimated between nodal pair points and then location of nodes will be computed inside the defined network. Range based methods include some important elements such as Time of Arrival (ToA), Received Signal Strength Indicator (RSSI), Time Difference of Arrival (TDoA) and Angle of Arrival (AoA). In fact, these are needed parameters in this method in order to acquire direction and distance of sensor nodes from reference points inside network.

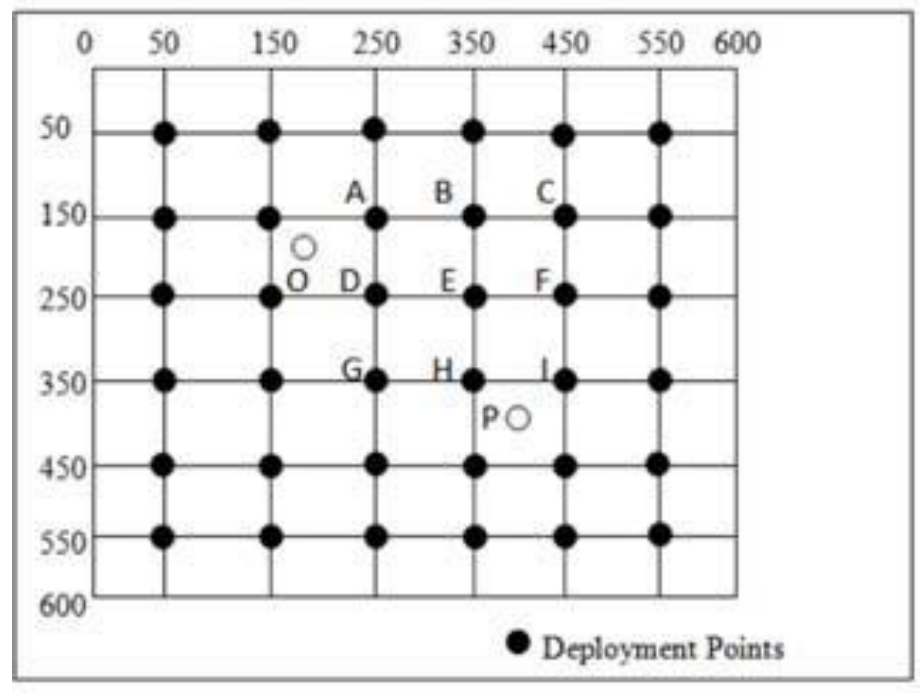

Figure 3. An illustrative example of using set of predefined deployment points

AOA is applied to evaluate angle that signals will be received from sensor nodes and then process of computing nodal locations by means of principles of geometric methods will be done. Based on RSSI, radios will be added to sensor nodes as transmitter and receiver. Also, by having main power from transmitter and compared to power from received signal, favorite distance is computed. In TOA and TDoA, this distance is computed based on propagation time parameter. Propagation time measures difference between sent and received time. Another defined parameter is Roundtrip propagation time. It calculates the difference in sent and received time of returned signal from original sensor. Also, the position of transmitter is estimated through TDoA[10].

Range free methods works according to connectivity of reference points and deploys locality information from number of nodes and its connectivity in order to find location of nodes and the distance parameter is not estimated based on mentioned locality nodes. Meanwhile, connectivity information will be deployed in order to estimate position of nodes and hence it is counted for a cost effective method due to not usage of any special hardware and sensor nodes work properly in a dense environment[11][12].

In centralized localization approach, for gathering information will be used a single processor and after that global map will be computed. Sensor nodes send information to a central node that can evaluate position of each sensor node and then it will be informed to nodes about their positions. Application of this method is more to control and monitor traffic of roads, environments and health issues[13][14][15][16][17].

In distributed localization method, position of each sensor node will be obtained independently and also localization of every node through the distance of that mentioned node will be measured and local information is identified through local neighbor nodes. 


\section{Conclusions and Discussion on Limitations}

In this survey, our aim has been to introduce and analyze optimized methods in order to find location and distance of sensor nodes in a defined network. Also, some of disadvantages of these methods have been reviewed in whole manuscript. Now, in order to come into conclusion, we point out to some issues. In these presented methods, it was not discussed about using a position of satellite in order to send local information and just it has been said that there is no need to any hardware. But, by extension of internet in each area that is connected to Global WWW network, the main problem is the subject of bandwidth and frequency that each satellite can cover local Wi-Fi networks that we considered it as a computational format by WSNs. Future defined networks that are called Li-Fi cover and support all needed items that issued to make a new hi-speed internet by more broadband and in order to solve previous mentioned problems. Although, in these new networks, researchers and network engineers try to find optimal wats in order to gain most facility from new designed and suggested network to all users and ISP companies that are going to present these new services to customers. Therefore, any new technology or existing defined WSNs have their own advantages and

\section{References}

[1] Samira Afzal, "A Review of Localization Techniques for Wireless Sensor Networks", Journal of Basic and Applied Scientific Research, Volume 2, Issue 8, pp. 7795-7801, September 2012.

[2] P.K Singh, Bharat Tripathi and Narendra Pal Singh, "Node Localization in Wireless Sensor Networks", International Journal of Computer Science and Information Technologies (IJCSIT), Volume 2, Issue 6, pp. 2568-2572, December 2011.

[3] Osamah Ibrahim Khalaf, Ghaida Muttashar Abdulsahib and Muayed Sadik, 2018. A Modified Algorithm for Improving Lifetime WSN. Journal of Engineering and Applied Sciences, 13: 9277-9282

[4] Prashant Tiwari, Varun Prakash Saxena, Raj Gaurav Mishra, Devendra Bhavsar, Wireless Sensor Networks: Introduction, Advantages, Applications and Research Challenges, HCTL Open International Journal of Technology Innovations and Research (IJTIR),http://ijtir.hctl.org, Volume 14, April 2015.

[5] Lili Yang, Optimising locations of sink nodes in wireless sensor networks using genetic algorithms, IEEE International Conference on Systems, Man \& Cybernetics (SMC), Taipei, Taiwan,2006, Feature+ ,Vol 39/7.

[6] Shikha, A Study for Finding Location of Nodes in Wireless Sensor Networks, International Journal of Computer Science \& Engineering Technology (IJCSET), Vol. 7 No. 03 Mar 2016.

[7] Ayman Dawood Salman1, Osamah Ibrahim Khalaf and Ghaida Muttashar Abdulsahib, 2019. An adaptive intelligent alarm system for wireless sensor network. Indonesian Journal of Electrical Engineering and Computer Science, Vol. 15, No. 1, July 2019, pp. 142 147

[8] Ogudo, K.A.; Muwawa Jean Nestor, D.; Ibrahim Khalaf, O.; Daei Kasmaei, H. A Device Performance and Data Analytics Concept for Smartphones' IoT Services and Machine-Type Communication in Cellular Networks. Symmetry 2019, 11, 593.

[9] Hager Ramadan, Ahmed M. khedr, Dharma P. Agrawal, “Effective data routing using mobile sinks in disjoint mobile wireless sensor networks", Periodicals of Engineering and Natural Sciences, Vol 7, No 3 (2019)

[10] GHAIDA MUTTASHAR ABDULSAHIB and OSAMAH IBRAHIM KHALAF, 2018. AN IMPROVED ALGORITHM TO FIRE DETECTION IN FOREST BY USING WIRELESS SENSOR NETWORKS.International Journal of Civil Engineering \& Technology (IJCIET) - Scopus Indexed.Volume:9,Issue:11,Pages:369-377.

[11] Osamah Ibrahim Khalaf, Ghaida Muttashar Abdulsahib and Muayed Sadik, 2018. A Modified Algorithm for Improving Lifetime WSN. Journal of Engineering and Applied Sciences, 13: 9277-9282

[12] N. Sulaiman, G. Abdulsahib, O. Khalaf, M. N. Mohammed, "Effect of Using Different Propagations of OLSR and DSDV Routing Protocols", Proceedings of the IEEE International Conference on Intelligent Systems Structureing and Simulation, pp. 540-545, 2014 
[13] Osamah Ibrahem Khalaf, Norrozila Sulaiman, Ghaidaa Muttasher Abdulsahib,2014.Analyzing Video Streaming Quality by Using Various Error Correction Methods on Mobile Ad hoc Networks in NS2", International Journal of Engineering Research and Applications . 4(10):pp.172-178.

[14] Anatoly N. Yakubovich, Yury V. Trofimenko, Irina A. Yakubovich, Elena V. Shashina, “ A forecast model for a road network's section traffic capacity assessment on a territory of the cryolithozone in conditions of the climate change", Periodicals of Engineering and Natural Sciences, Vol 7, No 1 (2019)

[15] Monalisa Mishra, Pradeep Kumar Singh, Aditya Brahmachari, Narayan C Debnath, Prasenjit Choudhury, “ A Robust Pest Identification System using Morphological Analysis in Neural Networks", Periodicals of Engineering and Natural Sciences, Vol 7, No 1 (2019)

[16] Olha Prokopenko, Vitaliy Omelyanenko, T. Ponomarenko, O. Olshanska, “ Innovation networks effects simulation models", Periodicals of Engineering and Natural Sciences, Vol 7, No 2 (2019)

[17] Kavitha V, “ Efficient and Optimal Routing Using Ant Colony Optimization Mechanism for Wireless Sensor Networks, Vol 6, No 1 (2018) 